\title{
Enhancing Hospital Well-being and Minimizing Intensive Care Unit Trauma: Cushioning Effects of Psychosocial Care
}

\author{
Usha Chivukula, Meena Hariharan, Suvashisa Rana, Marlyn Thomas, Asher Andrew \\ Centre for Health Psychology, University of Hyderabad, Hyderabad, Telangana, India
}

\section{Abstract}

Context: Hospitalization has the potential to induce hospital anxiety, while admission in the Intensive Care Unit (ICU) is found to surpass the anxiety and result in what is termed as "ICU Trauma." Aims: This study aimed to determine the impact of psychosocial care and quality of ICU on ICU trauma and hospital well-being in patients who underwent coronary artery bypass grafting (CABG). Settings and Design: This correlational study involved 250 CABG patients, who were recruited from five major corporate hospitals. Participants and Methods: The ICU Psychosocial Care Scale, Hospital Wellbeing Scale, and ICU Trauma Scale were used. Each of the participants was assessed individually. The ICU Practices Checklist was used to assess the environment of the ICU in the hospital. Statistical Analysis Used: Descriptive statistics, correlation, and simple and multiple linear regression analyses were done. Results: The results revealed the significant contribution of psychosocial care in ICU in enhancing hospital well-being as well as minimizing ICU trauma of patients who underwent CABG. The results of multiple regressions clearly indicated that psychosocial care was a powerful predictor of hospital well-being and ICU trauma. Conclusions: Although psychosocial care was not a component of hospital well-being and had a negative correlation with ICU trauma, it contributed significantly with a cushioning effect to minimize trauma and helped enhance the feelings and experiences of well-being among patients in ICU.

Keywords: Coronary artery bypass grafting, hospital well-being, Intensive Care Unit trauma, psychosocial care

\section{INTRODUCTION}

Hospitalization has the potential to induce hospital anxiety, while admission in the Intensive Care Unit (ICU) is found to surpass the anxiety and result in what is termed as "ICU Trauma." ICU trauma refers to a phenomenon resulting from a patient's stay in ICU of a hospital. It is the patient's strong emotional experience such as vulnerability, shock, intense fear, or emotional numbing, which in turn impacts the cognition and behavior of the patient manifested in the form of cognitive disorientation, avoidance behavior, and taking many other negative forms. Patients undergoing coronary artery bypass graft (CABG) surgery are required to stay in the ICU for 4-6 days during which they convalesce under close monitoring. The extreme environmental conditions of the ICU induce physical and mental stress and anxiety in the patient. The management of such distress in the ICU necessitates psychosocial care and intervention in addition to the ongoing medical treatment. The subsequent outcomes of such care can be measured on medical and nonmedical criteria. The nonmedical criterion is measured in terms of

\begin{tabular}{|l|l|}
\hline \multicolumn{3}{c|}{ Access this article online } \\
\hline Quick Response Code: & Website: \\
\hline & www.ijccm.org \\
\hline
\end{tabular}

hospital well-being which refers to the subjective perception and evaluation of a patient's health condition in terms of his/her affect states, psychological functioning, social relations, and spiritual well-being.

The ICU atmosphere is that of a large room, with artificial lightning, beeps of monitoring machines, sounds of ventilators, mourns of patients, constant movement of highly skilled doctors and nursing staff, and the absence of family. A stay in this environment impinges heavily upon patient's physical and psychological resources. A clinical review ${ }^{[1]}$ reported that weight loss, fatigue, poor appetite, and muscle weakness may impair physical functioning, which results in severe exhaustion such that patients are unable to accomplish even simple physical tasks. Tubes and wires attached to the body

Address for correspondence: Dr. Suvashisa Rana,

Centre for Health Psychology, University of Hyderabad, Central University Campus P. 0., Gachibowli, Hyderabad - 500 046, Telangana, India. E-mail: suvemail@gmail.com

This is an open access article distributed under the terms of the Creative Commons Attribution-NonCommercial-ShareAlike 3.0 License, which allows others to remix, tweak, and build upon the work non-commercially, as long as the author is credited and the new creations are licensed under the identical terms.

For reprints contact: reprints@medknow.com

How to cite this article: Chivukula U, Hariharan M, Rana S, Thomas M, Andrew A. Enhancing hospital well-being and minimizing intensive care unit trauma: Cushioning effects of psychosocial care. Indian J Crit Care Med 2017;21:640-5. 
immobilize patients which then increases dependency and vulnerability and adds to a feeling that they no longer are in charge of their physical condition. ${ }^{[2-4]}$ The extreme physical environment of the ICU additionally engenders alterations in sensory inputs, namely, sensory deprivation, sensory overload, excessive noise, physical and social isolation, and restriction of movement, which are some of the causes of psychological trauma. A review study ${ }^{[5]}$ on nocturnal care interactions in the ICU during 147 nights revealed that several nursing activities such as measuring vital signs, administering medication, obtaining blood samples, and bathing patients take place between $7 \mathrm{pm}$ and $6 \mathrm{am}$, thus requiring continuous light at the nursing station and near the patient's bed. Strong lightning and noise affect physiological parameters such as blood pressure, heart rate, and sleep. ${ }^{[6]}$ These stressors and ongoing activities contribute to the incidence of ICU delirium, i.e., a temporary loss of orientation in time and place and experience of unreal events. ${ }^{[7,8]}$ The experience of ICU patients is equated to that of a torture victim, ${ }^{[9]}$ claiming that debility and dependence in torture situations are produced by depriving people of food, drink, sleep, and human contact, and the same situation occurs in the ICU. Patients in ICU exhibit symptoms similar to posttraumatic stress disorder (PTSD). These reactions persist even after the ICU stay. The reactions appear as re-experiencing the event (flashbacks), avoiding situations that remind one of the events, a numbed reaction, and hyperarousal. ${ }^{[10]}$ Thus, the traumatic experiences of the ICU are long-lasting and have adverse effects on patients' cognitive-affective functioning. ${ }^{[1]}$ ICU patients showed strikingly high rates of psychological morbidity, which is claimed to be reduced using psychological interventions along with modified pharmacological and medical interventions. ${ }^{[12]}$

Consider also that the prevalence of anxiety and depression among cardiac patients is three times higher than in the general population, with $25 \%-30 \%$ of patients reporting persistent problems with anxiety and/or depression. ${ }^{[13]}$ In combination with ICU trauma, the patient's state may only worsen. Research reveals that the management of delirium is done mainly through pharmacological treatment. However, few nonpharmacological interventions such as exercise and early mobility are found to be helpful in reducing delirium. An interventional study on early mobility and reduction of delirium in ICU patients ${ }^{[14]}$ found that the group of patients who were given physical and occupational therapy along with the interruption of sedation were found to have a significant decrease in delirium (50\%) in the ICU. Nonetheless, several studies on cardiac patients ${ }^{[15-18]}$ noted that interventions such as guided imagery, relaxation techniques, emotional and informational support, personal attention by medical professionals, and breathing exercises helped reduce the impact of the ICU environment and enhanced patient outcomes. Evidence that multiple psychosocial factors may influence adaptation to heart surgery has implications for understanding and ameliorating presurgical distress and for improving postsurgical recovery. ${ }^{[19]}$ Research has furthermore revealed that psychological preparation, ${ }^{[20]}$ cognitive interventions such as preoperative information ${ }^{[21]}$ and cardiac rehabilitation ${ }^{[22]}$ can improve patient outcomes and reduce emotional distress. If postsurgery interventions and psychological preparation can improve the patient's well-being, then a strategically planned psychosocial care intervention in the ICU can have a greater impact on the overall patient outcomes. Consequently, an ICU which fosters psychosocial support can accelerate patients' holistic well-being and recovery.

National and international boards such as the National Accreditation Board for Hospitals (NABH), ${ }^{[23]}$ the Joint Commission International (JCI), ${ }^{[24]}$ and the Society of Critical Care Medicine (SCCM) ${ }^{[25]}$ provide guidelines and set standards for hospitals to ensure holistic care for patients. Although psychosocial care in the health-care system is insisted upon by these guidelines, it has not received the attention and the significance it deserves. Moreover in India, the practices followed in hospitals are skewed toward the biomedical model, leaving aside psychosocial care. This brings us to a question whether adherence, partial adherence, or nonadherence to these standard guidelines for psychosocial care results in significant differences in hospital well-being and ICU trauma? Hence, the study was conceptualized to determine the impact of psychosocial care and ICU quality on ICU trauma and hospital well-being in patients who were admitted in cardiac ICUs for a stipulated period of time after CABG surgery.

\section{Participants and Methods}

\section{Participants}

The correlational study using a multistage sampling included 250 patients in the sample who were moved into the ward after a stay of 4-6 days in the ICU after undergoing CABG. The participants were a convenience sample selected from five corporate hospitals. From each hospital, fifty participants were selected. The participants included both men (69\%) and women (31\%). Their age ranged from 40 to 75 years, with a mean age of 55 years (standard deviation $=9.7$ ). The inclusion criteria for hospitals and the sample were as follows.

\section{Inclusion criteria for hospitals}

We approached hospitals that were accredited by NABH, where at least $12 \mathrm{CABG}$ surgeries per week were performed and that were willing to sign the informed consent form.

\section{Inclusion criteria for patients}

All patients aged between 40 and 75 years who underwent CABG with a minimum stay of 2 days in the ICU, who were in a condition to communicate verbally, and who were willing to participate in the study and signed the informed consent form were included in the patient sample.

\section{Instruments}

We used the ICU Psychosocial Care Scale, Hospital Wellbeing Scale, ICU Trauma Scale, and ICU Practices Checklist. These tools are described below. 


\section{The Intensive Care Unit psychosocial care scale}

The scale was a revised version of Intensive Care Experience Rating Scale ${ }^{[1]}$ and consisted of 18 items, each measured with a 5-point rating scale ranging from "Never" to "Always." The scale measured psychosocial care in three dimensions, namely, protection of human dignity with the dimension score ranging between 7 and 35, family-patient communication channel, where the dimension score ranged between 6 and 30, and family-patient anxiety prevention with the dimension scores ranging from 5 to 25 . The total score ranged from 18 to 90 . Cronbach's alpha was found to be 0.75 in the present sample.

\section{The Hospital Wellbeing scale}

The Hospital Wellbeing Scale was constructed taking the Warwick-Edinburgh Mental Wellbeing Scale ${ }^{[26]}$ as the basis. It measured four important dimensions of health, i.e., physical, mental, social, and spiritual health using 28 items. The scale measured the feelings and experiences of the patients by assessing how often the patient feels the way described in the item on a 5-point scale ranging from "None of the time" to "All of the time." Sum of the items on each dimension was the score for that particular dimension. The maximum score for the scale was 140 and the minimum score was 28 . Since each dimension comprised of 7 items, the scoring for each of the dimensions, namely, psychological, physical, social, and spiritual well-being ranged between 7 and 35 . Cronbach's alpha was found to be 0.88 in the present sample.

\section{The Intensive Care Unit trauma scale}

The scale was a modified version of Davidson Trauma Scale. ${ }^{[27]}$ It consisted of 15 items measuring three dimensions of trauma, namely, re-experience, emotional numbing and avoidance, and hyperarousal on a 5-point rating scale ranging from "Not at all" to "Always." The re-experience dimension had 5 items where the dimension scores ranged from 5 to 25 . There were six items in emotional numbing and avoidance with the dimension scores ranging from 6 to 30 . Four items measured hyperarousal that ranged the scores from 4 to 20 . The overall trauma score ranged from 15 to 75 . Cronbach's alpha was found to be 0.72 in the present sample.

\section{The Intensive Care Unit practices checklist}

The ICU Practices Checklist was used to measure ICU quality. This scale was developed based on the guidelines for maintaining standards of ICUs provided by various bodies such as the JCI, the Indian SCCM, and the NABH. The checklist consisted of 34 statements related to ICU practices. This was used by the investigators who checked the presence or absence of the practices stated in the checklist. The scores ranged between 0 and 34 .

\section{Procedure}

After completing the administrative formalities and informed consent, the patients were contacted in the ward/rooms within $24 \mathrm{~h}$ of shifting them from ICU. The scales were administered on them. Sometimes, when they expressed tiredness, the administration of tools was staggered to suit their convenience.

The ICU Quality Checklist was used by the first author who observed each of the five ICUs at different points of time of the day and night.

\section{RESULTS}

The results attempted to identify different factors positively contributing to hospital well-being and also those factors that contribute to minimize ICU trauma. Separate simple linear regression analyses were carried out for hospital well-being and ICU trauma. The independent variables for both the criterion variables were psychosocial care, ICU quality, income level, age, duration of stay (in the hospital), and gender. The findings of simple linear regression analyses are summarized in Table 1.

It can be observed from Table 1 that, with regard to hospital well-being, among all the predictors, psychosocial care explained statistically significant $(18 \%)$ proportion of the variance, $R^{2}=0.18$ and adjusted $R^{2}=0.18, F(1,248)=56.80$, $P<0.01$. The relationship between psychosocial care and well-being was positive, $\beta=0.43, P<0.01$, showing that high psychosocial care was associated with high well-being. The high $\beta$-value shows a strong relation between the predictor psychosocial care and criterion, i.e., hospital well-being. The results in addition showed that income level was also a significant predictor of well-being, though it accounted for only $2 \%$ of variance on the well-being scores, $R^{2}=0.02$, adjusted $R^{2}=0.01, F(1,248)=5.2, P<0.05$. A positive correlation between income level and hospital well-being was observed $(\beta=0.15$ and $P<0.05)$.

Table 1: Summary of simple regression analyses for variables predicting hospital well-being and Intensive Care Unit trauma in Intensive Care Unit patients who underwent coronary artery bypass grafting

\begin{tabular}{|c|c|c|c|c|c|c|c|c|c|c|c|c|}
\hline \multirow[t]{2}{*}{ Predictors } & \multicolumn{6}{|c|}{ Hospital well-being } & \multicolumn{6}{|c|}{ ICU trauma } \\
\hline & $B$ & SEB & $\boldsymbol{\beta}$ & SE & $R^{2}$ & $F(1,248)$ & B & SEB & $\boldsymbol{\beta}$ & SE & $R^{2}$ & $F(1,248)$ \\
\hline Psychosocial care & 0.81 & 0.11 & $0.43 * *$ & 11.43 & 0.18 & $56.80 * *$ & -0.41 & 0.07 & $-0.34 * *$ & 7.76 & 0.12 & $32.80^{* *}$ \\
\hline ICU quality & 0.23 & 0.22 & 0.06 & 12.64 & 0.004 & 1.09 & -0.30 & 0.15 & $-0.13^{*}$ & 8.19 & 0.02 & $4.13^{*}$ \\
\hline Income level & 2.2 & 0.98 & $0.15^{*}$ & 12.54 & 0.02 & $5.2^{*}$ & -1.13 & 0.64 & -0.11 & 8.21 & 0.01 & 3.15 \\
\hline Age & 0.06 & 0.08 & 0.05 & 12.65 & 0.003 & $<1$ & 0.04 & 0.05 & 0.05 & 8.25 & 0.01 & $<1$ \\
\hline Duration of stay & 1.18 & 0.91 & 0.08 & 12.63 & 0.007 & 1.7 & -0.07 & 0.59 & -0.01 & 8.26 & 0.01 & $<1$ \\
\hline Gender & 1.73 & 1.73 & 0.06 & 12.64 & 0.004 & $<1$ & 1.88 & 1.13 & 0.10 & 8.21 & 0.01 & 2.73 \\
\hline
\end{tabular}

$n=250 ; * P<0.05 ; * * P<0.01 . B$ : Unstandardized beta coefficient; SEB: Standardized error of beta; $\beta$ : Standardized beta coefficient; SE: Standard error of the estimates; ICU: Intensive Care Unit 
The findings of simple linear regression analyses for the criterion ICU trauma revealed that statistically significant $(11 \%)$ proportion of the variance in ICU trauma was explained by psychosocial care, $R^{2}=0.12$, adjusted $R^{2}=0.11$, $F(1,248)=32.80, P<0.01$. The relationship between psychosocial care and ICU trauma was negative, $\beta=-0.34$, $P<0.01$, stating that, with increase in the psychosocial care, the ICU trauma among patients decreased. Apart from the psychosocial care, ICU trauma was also caused by the very environment of the ICU vis-à-vis the physical condition of the patient. The results of simple linear regression analysis showed that the ICU quality had an impact on ICU trauma. A small yet a statistically significant (2\%) proportion of the variance in ICU trauma was explained by ICU quality, $R^{2}=0.02$, adjusted $R^{2}=0.01, F(1,248)=4.13, P<0.05$. ICU quality was inversely related to ICU trauma, $\beta=-0.13 P<0.05$, i.e., improved ICU quality results in reduced ICU trauma.

The findings infer that psychosocial care and good ICU quality played a positive role in mitigating or minimizing ICU trauma. Evidence from literature associated psychological distress and trauma as a consequence of ICU stay. Studies have recommended the need of psychological approaches and psychosocial interventions in dealing with ICU trauma. Such interventions can be brought about only by enhancing psychosocial care. The results of simple linear regression analyses substantiated the above statement by indicating that psychosocial care and ICU quality were significant contributors in reducing ICU trauma, while variables such as age, gender, duration of stay in hospital, and income level played no significant role in determining ICU trauma.

Thus, two variables - psychosocial care and income level - were found to independently contribute to hospital well-being while two variables - psychosocial care and ICU quality - were found to independently contribute to ICU trauma. Carrying it forward, two separate multiple linear regression analyses using simultaneous method were conducted to find the combined effect of psychosocial care and income level on hospital well-being and the combined contribution of psychosocial care and ICU quality on ICU trauma. The results are presented in Table 2.

Simultaneous multiple linear regression analysis for hospital well-being presented in Table 2 revealed that the combined predictors of psychosocial care and income level explained $19 \%$ of variance in hospital well-being, $R^{2}=0.19$, adjusted $R^{2}=0.18, F(2,247)=28.74, P<0.01$. Psychosocial care was the only significant predictor $(\beta=0.45, P<0.01)$, whereas income level was not found to be a significant predictor ( $\beta=0.06, P>0.05)$ in the final model.

As it can be observed from Table 2, ICU trauma in patients was significantly negatively related, $\beta=-0.38, P<0.01$, to psychosocial care showing that higher levels of psychosocial care are associated with lower levels of ICU trauma. The multiple linear regression revealed that the combined predictors of psychosocial care and ICU quality explained

\begin{tabular}{|c|c|c|c|c|c|c|}
\hline \multirow[t]{2}{*}{ Predictors } & \multicolumn{3}{|c|}{ Hospital well-being } & \multicolumn{3}{|c|}{ ICU trauma } \\
\hline & $B$ & SEB & $\beta$ & $B$ & SEB & $\boldsymbol{\beta}$ \\
\hline Psychosocial care & 0.85 & 0.12 & $0.45 * *$ & -0.47 & 0.09 & $-0.38 * *$ \\
\hline Income level & 0.85 & 0.99 & 0.06 & - & - & - \\
\hline ICU quality & - & - & - & 0.20 & 0.17 & $-0.08^{*}$ \\
\hline$R^{2}$ & \multicolumn{3}{|c|}{0.19} & \multicolumn{3}{|c|}{0.12} \\
\hline Constant & \multicolumn{3}{|c|}{44.43} & \multicolumn{3}{|c|}{67.32} \\
\hline$F(2,247)$ & \multicolumn{3}{|c|}{$28.74 * *$} & \multicolumn{3}{|c|}{$17.14^{* *}$} \\
\hline
\end{tabular}

$n=250 ; * P<0.05 ; * * P<0.01 . B$ : Unstandardized beta coefficient; SEB: Standardized error of beta; $\beta$ : Standardized beta coefficient; ICU: Intensive Care Unit

$12 \%$ of variance in ICU trauma, $R^{2}=0.12$, adjusted $R^{2}=0.12, F(2,247)=17.14, P<0.01$. It can also be observed from Table 2 that ICU quality was also negatively correlated, $\beta=-0.08, P<0.05$ with ICU trauma, showing that higher ICU quality was associated with lower levels of ICU trauma. Thus, both psychosocial care and ICU quality were found to be the significant predictors of ICU trauma.

The results of simple linear regression and multiple linear regression analyses showed that, while psychosocial care was a significant predictor of ICU trauma, the influence of ICU quality on ICU trauma was marginal. From the simple and multiple regression analyses to predict hospital well-being and ICU trauma, it is very evident that psychosocial care emerged as a significant predictor of hospital well-being and ICU trauma, while the contributions of income level to hospital well-being and the ICU quality to ICU trauma were marginal. Psychosocial care thus emerged as a significant major contributor in enhancing hospital well-being and minimizing ICU trauma.

The results of linear regression analyses revealed that a significantly higher degree of psychosocial care was found to have not only a positive impact on hospital well-being, but also minimize ICU trauma. This in turn is expected to have a noticeable influence on the pace of recovery, restoration of well-being, and minimizing the chances of re-hospitalization.

\section{Discussion}

The present study was taken up to examine if psychosocial care has a cushioning effect in enhancing hospital well-being and minimizing, if not, mitigating ICU trauma. The results clearly indicate the significant contribution of psychosocial care in ICU in enhancing hospital well-being as well as minimizing ICU trauma of patients who undergo CABG. The results of multiple regressions clearly indicated that psychosocial care is a powerful predictor of hospital well-being and ICU trauma.

Clinician-patient relationship is extremely vital in a critical care setting. ${ }^{[28]}$ Supportive interventions, such as explanations, 
giving advice, reassuring and raising faith and hope, strengthening patients' self-esteem, giving emotional warmth, empathetic listening and empathetic touch, emotional care, and spending extra time with patients are some of the psychological and social aspects. ${ }^{[29]}$ Such interventions have a cushioning effect on the patient by providing the cushion for absorbing the adverse impact of ICU and minimize ICU trauma. Further, the cushion of psychosocial care also helps in springing up the well-being in patients.

ICU care that does not include the above aspects leaves the patient anxious, disoriented, uncertain, vulnerable, unattended, and perhaps unventilated and suppressed. This has its repercussion in the form of nightmares, flashbacks, re-experience, avoidance, and numbing culminating in trauma that sometimes closely resembles PTSD even after their discharge from ICU. The results of the present study corroborate with earlier findings that negative emotions, when intervened with psychosocial care, can prevent the immediate- and long-term negative impact. Negative emotions and stress have both immediate- and long-term effects on patients' physical and psychological well-being. ${ }^{[30]}$ Providing psychosocial care helps reduce negative stress and restores well-being. The results of the study highlighted that patients who received high psychosocial care had higher levels of well-being and lower levels of ICU trauma. It is very clear that, though not a component of hospital well-being, psychosocial care helps in enhancing the feelings and experiences of well-being among patients in ICU. In the same manner, though independent of ICU trauma, it is found to have a negative correlation with ICU trauma and significantly contribute in minimizing trauma. Thus, the role of psychosocial care is that of a medium which influences ICU trauma and hospital well-being.

It is important for the hospitals to reorient themselves on psychosocial care in view of its great contribution in mitigating ICU trauma and enhancing well-being. Research has proved that trauma negatively impacts convalescence, ${ }^{[31]}$ and sometimes is responsible for relapse and rehabilitation. ${ }^{[32]}$ Psychosocial care functions as prophylactic in preventing such repercussions of ICU trauma.

\section{Conclusion}

Basically psychosocial care relates to the attitude and behavior of the middle-level workers, such as nursing staff and attendants. Psychosocial care relates to some extent to the ethical practices, such as protecting the patient's privacy, obtaining oral informed consent before procedures, explaining the procedures before initiating so as to minimize, if not mitigate the anxiety, responding to nonverbal communication of the patient, and liaisoning between the patient and family. These practices do not call for specific allocation of time or funds. This can be introduced, incorporated, monitored, and measured by introducing regular in-service training for the staff where they should be oriented and sensitized to the psychosocial needs of the patients. This minor intervention through a policy by the management of hospitals would be highly beneficial both to the patient and to the management in view of the pace of recovery for the patient and reputation of the hospital. A higher level of psychosocial care which demands a holistic approach and a marginal increase in time invested in communication may prove to be cost-effective when assessed for the impact on the patients' well-being. Hence, it calls for the hospitals to weigh its overall advantages and include it as an important dimension in ICU care.

\section{Acknowledgment}

We acknowledge the active participation of the participants, doctors, and nurses during the entire phase of our study.

\section{Financial support and sponsorship}

Nil.

\section{Conflicts of interest}

There are no conflicts of interest.

\section{RefERENCES}

1. Broomhead LR, Brett SJ. Clinical review: Intensive care follow-up - What has it told us? Crit Care 2002;6:411-7.

2. Almerud S, Alapack RJ, Fridlund B, Ekebergh M. Caught in an artificial split: A phenomenological study of being a caregiver in the technologically intense environment. Intensive Crit Care Nurs 2008;24:130-6.

3. Gjengedal E. Understanding a World of Critical Illness. A Phenomenological Study of Experiences of Respirator Patients and Their Caregivers [Dissertation] University of Bergen; 1994.

4. Samuelson K. Sedation during Mechanical Ventilation in Intensive Care: Sedation Practices and Patients' Memories, Stressful Experiences and Psychological Distress. [Dissertation] Lund University Sweden; 2006.

5. Tamburri LM, DiBrienza R, Zozula R, Redeker NS. Nocturnal care interactions with patients in critical care units. Am J Crit Care 2004; 13:102-12.

6. Ryherd EE, Waye KP, Ljungkvist L. Characterizing noise and perceived work environment in a neurological critical care unit. J Acoust Soc Am 2008;123:747-56.

7. Granberg-Axèll A, Bergbom I, Lundberg D. Clinical signs of ICU syndrome/delirium: An observational study. Intensive Crit Care Nurs 2001;17:72-93.

8. Granberg Axèll AI, Malmros CW, Bergbom IL, Lundberg DB. Intensive Care Unit syndrome/delirium is associated with anemia, drug therapy and duration of ventilation treatment. Acta Anaesthesiol Scand 2002;46:726-31.

9. Dyer I. Preventing the ITU syndrome or how not to torture an ITU patient! Part I. Intensive Crit Care Nurs 1995;11:103-39.

10. Margolies L. Understanding the Effects of Trauma: Post-Traumatic Stress Disorder (PTSD). Psych Central; 2010. Available from: http:// www.psychcentral.com/lib/understanding-the-effects-of-trauma-posttraumatic-stress-disorder-ptsd/0003971. [Last accessed on $2013 \mathrm{Jul} 22$ ].

11. Hariharan M, Chivukula U. Patient care in Intensive Care Units (ICUs): Biopsychosocial assessment. J Indian Health Psychol 2011;5:25-35.

12. Wade DM, Howell DC, Weinman JA, Hardy RJ, Mythen MG, Brewin CR, et al. Investigating risk factors for psychological morbidity three months after intensive care: A prospective cohort study. Crit Care 2012;16:R192.

13. Blumenthal JA, Lett HS, Babyak MA, White W, Smith PK, Mark DB, et al. Depression as a risk factor for mortality after coronary artery bypass surgery. Lancet 2003;362:604-9.

14. Schweickert WD, Pohlman MC, Pohlman AS, Nigos C, Pawlik AJ, Esbrook CL, et al. Early physical and occupational therapy in 
mechanically ventilated, critically ill patients: A randomised controlled trial. Lancet 2009;373:1874-82.

15. Bergmann P, Huber S, Mächler H, Liebl E, Hinghofer-Szalkay H, Rehak $\mathrm{P}$, et al. The influence of medical information on the perioperative course of stress in cardiac surgery patients. Anesth Analg 2001;93:1093-9.

16. Friesner SA, Curry DM, Moddeman GR. Comparison of two pain-management strategies during chest tube removal: Relaxation exercise with opioids and opioids alone. Heart Lung 2006;35:269-76.

17. Halpin LS, Speir AM, CapoBianco P, Barnett SD. Guided imagery in cardiac surgery. Outcomes Manag 2002;6:132-7.

18. Hwang SL, Chang Y, Ko WJ, Lee MB. Stress-reducing effect of physician's tape-recorded support on cardiac surgical patients in the Intensive Care Unit. J Formos Med Assoc 1998;97:191-6.

19. Contrada RJ, Boulifard DA, Hekler EB, Idler EL, Labouvie EW, Spruill TM, et al. Psychosocial factors in heart surgery: Pre-surgical vulnerability and postsurgical recovery. Health Psychol 2008;7:309-19.

20. Currid T. Meeting the psychological needs of physically ill. Nurs Times 2012;108:22-5.

21. Arthur HM, Daniels C, McKelvie R, Hirsh J, Rush B. Effect of a preoperative intervention on preoperative and postoperative outcomes in low-risk patients awaiting elective coronary artery bypass graft surgery. A randomized, controlled trial. Ann Intern Med 2000;133:253-62.

22. Mampuya WM. Cardiac rehabilitation past, present and future: An overview. Cardiovasc Diagn Ther 2012;2:38-49.

23. National Accreditation Board for Hospitals \& Healthcare Providers. NABH Accredited Hospitals; 2012. Available from: http://www.nabh. $\mathrm{co} / \mathrm{main} / \mathrm{hospitals} /$ accredited.asp. [Last accessed on $2013 \mathrm{Jul}$ 04].

24. Joint Commission International Accreditation Standards for Hospitals.
Joint Commission International. $4^{\text {th }}$ ed. USA; 2010. Available from: http://www.jointcommissioninternational.org. [Last accessed on 2013 Jun 22].

25. Thompson DR, Hamilton DK, Cadenhead CD, Swoboda SM, Schwindel M, Anderson DC, et al. Guidelines for Intensive Care Unit design. Crit Care Med 2012;40:1586-600.

26. Tennant R, Hiller L, Fishwick R, Joseph S, Weich S, Stewart-Brown S. Warwick Edinburg Mental Wellbeing Scale (WEMWS): Development and UK validation. Health Qual Life Outcomes 2007;5:63.

27. Davidson JR, Book SW, Colket JT, Tupler LA, Roth S, David D, et al. Assessment of a new self-rating scale for post-traumatic stress disorder. Psychol Med 1997;27:153-60.

28. Wilkin K, Slevin E. The meaning of caring to nurses: An investigation into the nature of caring work in an Intensive Care Unit. J Clin Nurs 2004;13:50-9.

29. Frazier SK, Moser DK, O'Brien JL, Garvin BJ, An K, Macko M, et al. Management of anxiety after acute myocardial infarction. Heart Lung 2002;31:411-20

30. Deja M, Denke C, Weber-Carstens S, Schröder J, Pille CE, Hokema F, et al. Social support during Intensive Care Unit stay might improve mental impairment and consequently health-related quality of life in survivors of severe acute respiratory distress syndrome. Crit Care 2006;10:R147.

31. Barskova T, Oesterreich R. Post-traumatic growth in people living with a serious medical condition and its relations to physical and mental health: A systematic review. Disabil Rehabil 2009;31:1709-33.

32. Hudetz JA, Gandhi SD, Iqbal Z, Patterson KM, Byrne AJ, Warltier DC, et al. History of post-traumatic stress disorder is associated with impaired neuropsychometric performance after coronary artery surgery. J Cardiothorac Vasc Anesth 2010;24:964-8. 